\title{
Costo de los accidentes de moto en menores de 19 años atendidos en un hospital de referencia
}

\section{Cost of motorcycle accidents in children under 19 years of age treated at a tertiary hospital}

\author{
Luis Báez', Doris Vergara de Navarro', Laura Heisele , Natalia Gómez ${ }^{1}$, Mirian Torales de \\ Cardozo ${ }^{1}$, Celia Martínez de Cuellar ${ }^{1}$
}

\section{RESUMEN}

Introducción: Los accidentes de tránsito constituyen un grave problema de salud pública, sin embargo, su impacto socioeconómico no se conoce en el Paraguay. Objetivos: determinar el impacto socioeconómico de los accidentes de moto en < 19 años en el año 2016. Materiales y Métodos: estudio descriptivo, observacional, retrospectivo. Se incluyeron todos los pacientes $\leq 19$ años que acudieron al Hospital de Trauma (HT) con antecedente de accidente de moto en el año 2016. Los datos y los costos fueron obtenidos de la base de datos del Dpto. de Estadísticas y del Dpto. de Ingresos del HT, respectivamente. Resultados: se incluyeron 700 pacientes que acudieron al HT por accidente de moto. La edad media de los pacientes fue de 15,6 \pm 4.33 años. El 78\% (546/700) era de sexo masculino. De los accidentados $450(64,2 \%)$ era conductor y $250(35,7 \%)$ acompañante. La media de edad de los conductores fue de $17 \pm 4,3$ años (rango $11-19$ años) y de los acompañantes $12,3 \pm 4,4$ (rango $<1-19$ años). Entre los conductores solo el 50.4\% (227/450) tenía la edad reglamentaria para obtener licencia de conducir. Solo el $7,7 \%(54 / 695)$ de los accidentados usaba casco al momento del accidente. Requirió hospitalización por 2 o más días el $89,2 \%(625 / 700)$ de los pacientes, de los cuales el $7.6 \%$ requirió ser hospitalizado en UCIP. La media de días de hospitalización en sala y UCIP fue de 11.04 días (DS 14,85 días) y 16.57 (DS 23 días). El 25,1\% (176/700) tuvo algún grado de discapacidad o secuela y el 5,4\% (38/700) falleció. Los costos directos por hospitalización por día en sala y en UCIP fueron de 275,4 y 737,2 \$, respectivamente. Los costos directos por accidentes de moto en el año 2016 fue de 2.587.179,2\$. Los años de vida potencialmente perdidos (AVPP) en el año 2016, fueron de 2159 años. Conclusiones: El impacto socioeconómico de los accidentes de motos, representa un costo social extremo

\section{ABSTRACT}

Introduction: Traffic accidents are a serious public health problem, however, their socio-economic impact has not been described in Paraguay. Objectives: to determine the socioeconomic impact of motorcycle accidents in victims $<19$ years of age in 2016. Materials and Methods: this was a descriptive, observational, retrospective study. All patients aged $\leq 19$ years who were admitted to the Trauma Hospital (TH) with a history of a motorcycle accident in 2016 were included. The data and costs were obtained from the Statistics Department and the Admissions Department of the TH. Results: 700 patients were included, all of whom were admitted to the $\mathrm{TH}$ due to a motorcycle accident. The average age of the patients was $15.6 \pm 4.33$ years. $78 \%(546 / 700)$ were male. Of those injured, $450(64.2 \%)$ were drivers and $250(35.7 \%)$ were passengers. The average age of the drivers was $17 \pm 4.3$ years (range 11-19 years) and that of the passengers was $12.3 \pm 4.4$ years (range $<1-19$ years). Among the drivers, only $50.4 \%(227 / 450)$ were of the legal age to obtain a driver's license. Only 7.7\% (54/695) of the victims wore a helmet at the time of the accident. $89.2 \%$ (625/700) of patients required hospitalization stays of 2 or more days of length, of which $7.6 \%$ required PICU admission. The mean number of regular ward hospitalization days and PICU days were 11.04 days (SD 14.85 days) and 16.57 days (SD 23 days), respectively. $25.1 \%$ (176/700) had some degree of disability or long-term effects and 5.4\% (38/700) died. The direct costs per hospitalization per day in the ward and in the PICU were US\$ 275.4 and 737.2, respectively. The direct costs for motorcycle accidents in 2016 was US\$ 2,587,179.2. The years of potential life lost (YPLL) in 2016 were 2159 years. Conclusions: The socioeconomic impact of motorcycle accidents represents an extremely high social cost, stemming from a preventable cause of death.

${ }^{1}$ Hospital de Trauma "Manuel Giagni". Asunción, Paraguay.

Correspondencia: Luis Carlos Báez; E-mail: lubaeza70@yahoo.es

Conflicto de Interés: Los autores declaran no poseer conflicto de interés

Recibido: 27/12/2017 Aceptado: 24/07/2018

DOI: https://doi.org/10.31698/ped.45022018005 
derivado de una causa de muerte que podría prevenirse.

Palabras claves: Accidente de motocicleta, muerte prematura, años de vida potencialmente perdidos. (AVPP)

\section{INTRODUCCIÓN}

Según la Organización Mundial de la Salud (OMS), los accidentes de tránsito causan anualmente, la muerte de aproximadamente 1,25 millones de personas en el mundo. Las lesiones causadas por el tránsito son la causa principal de muerte en el grupo de 15 a 29 años de edad. A pesar de que los países de ingresos bajos y medianos tienen aproximadamente la mitad de los vehículos del mundo, se producen en ellos más del $90 \%$ de las muertes relacionadas con accidentes de tránsito. La mitad de las personas que muere por esta causa son usuarios vulnerables de la vía pública; es decir, peatones, ciclistas y motociclistas $^{(1)}$.

Cada cuatro minutos un niño muere prematuramente en alguna carretera del mundo. Otros cientos sufren traumatismos, muchos de ellos graves. Estos hechos dolorosos provocan sufrimientos y penurias inconmensurables $y$, en ocasiones, dificultades económicas para las familias y los amigos. Además, suponen un costo para los valiosos recursos que las sociedades deben desviar de otros acuciantes problemas de salud y desarrollo ${ }^{(2)}$.

Los accidentes representan una importante causa de morbilidad y mortalidad a cualquier edad, y la población pediátrica no es la excepción. Los accidentes no respetan edad, sexo, raza o condición socioeconómica. A pesar de que es un acuerdo universal que los niños tienen el derecho a vivir en un ambiente seguro y a la protección contra lesiones y violencia, las lesiones en niños siguen siendo un problema de salud pública en todo el mundo. La OMS estima que mueren, en todo el mundo, aproximadamente 100 niños cada hora a causa de lesiones, de las cuales el $90 \%$ no es intencional ${ }^{(3)}$. En el Perú, de acuerdo a los datos de la Policía Nacional de Perú, durante los años 1998 al 2007 se han
Keywords: Motorcycle accident, premature death, years of potential life lost (YPLL)

registrado 405167 accidentes de tránsito, de los cuales 350.782 corresponden a heridos y ocasionaron la muerte de 32.105 personas, lo que evidencia el impacto de los siniestros producidos ${ }^{(4)}$.

Según datos del Ministerio de Salud, en el Paraguay, las muertes por accidentes de tránsito prácticamente se duplicaron en los últimos 10 años, aumentando de 9/10.000 en el 2001 a 19/10.000 en el 2010. Este incremento ocurre principalmente desde el 2006 y está relacionado con el crecimiento del número de motocicletas y ciclomotores en circulación, con el consecuente aumento de siniestros viales producidos por los mismos, constituyendo el $41 \%$ de los casos. La tendencia de las victimas lesionadas en eventos del tránsito, presenta un aumento a partir de la aparición de motocicletas en el país, llegando a la cifra de 40.375 personas en el año 2013. Hecho preocupante pues afecta extraordinariamente la respuesta de los servicios de salud y produce efectos socio económicos importantes para el país.

Se estima que los costos anuales de los accidentes de tránsito en los países de ingresos bajos y medios oscilan entre los 65 y los 100 mil millones de dólares, que es más que el monto total anual recibido para la ayuda al desarrollo ${ }^{(5)}$. Por otro lado, los mismos representan anualmente, entre 1 y $1,5 \%$ del producto nacional bruto en estos países, lo que ejerce una gran presión sobre los presupuestos de atención de la salud $^{(6)}$.

En España, se estima que los costos de la atención hospitalaria se encuentran en aproximadamente 565 euros por paciente, totalizando entre 243,435 y 598,422 euros anuales y para los denominados costos humanos valores que van de 105,687 y 258,661 euros respectivamente ${ }^{(7)}$. Por otro lado, en Argentina, el costo medio de tratamiento por 
atención de lesionados fue de $\$ 829$ según la variante de precios mínimos y los costos totales por accidentes de tránsito ocurridos en la Ciudad de Buenos Aires, en el año 2003 ascienden a \$54.874010, de los cuales 42.473.922, corresponden a costos indirectos por pérdidas de productividad y $\$ 12.400 .088$ son costos de atención sanitaria. Asimismo, reportan que los Años de vida saludables perdidas (APP) por año son de 4.148; de los cuáles el $86,3 \%$ corresponde a muerte prematura ${ }^{(8)}$.

En el Paraguay el grupo de víctimas más vulnerable involucrado en los accidentes son los jóvenes en el rango etario de 15 a 34 años (55\%) y en caso de peatones también los de más de 65 años. Otro grupo vulnerable en nuestro país son las victimas de siniestros viales donde se encuentran involucradas las motocicletas, y el grupo etario más afectado es el de 15 a 29 años ${ }^{(9)}$.

Según datos de fallecidos en el año 2015 en el Hospital del Trauma, los afectados llegan a su punto máximo en el rango de edad comprendido entre los 15 - 19 años, donde llega al 17\% del total.En el año 2016, en el Hospital de trauma Manuel Giagni fallecieron 447 personas, 165 fallecieron por siniestros en moto (37\%), 36 eran menores de 19 años $(22 \%)^{(10)}$.

En muchos países de ingresos bajos y medios, las motocicletas y bicicletas son un medio de transporte cada vez más común, los conductores de motociclistas constituyen una importante proporción del total de conductores lesionados o que mueren en las vías de tránsito. Los mismos están expuestos a mayor riesgo de colisiones porque comparten el espacio de circulación con vehículos de mayor porte que se desplazan velozmente y porque son menos visibles. Además, la falta de protección física los hace particularmente vulnerables a sufrir lesiones en el caso de una colisión ${ }^{(11)}$.

La determinación del impacto socioeconómico de los accidentes de tránsito en el Paraguay constituirá un insumo básico para la asignación equitativa de recursos a nivel nacional, de modo que los accidentes de tránsito sean calificados en una escala justa en relación a otros problemas de salud.Por lo cual, con este estudio se pretende caracterizar los accidentes de moto en $\leq 19$ años de pacientes que acuden al Hospital de Trauma Manuel Giagni así como determinar el costo de los accidentes de moto en $\leq 19$ años en el año 2016.

\section{MATERIALES Y MÉTODOS}

Se realizó un estudio descriptivo, observacional, retrospectivo. Se incluyeron todos los pacientes $\leq 19$ años que acudieron al Hospital de Trauma (HT) con antecedente de accidente de moto, ya sean conductores o acompañantes, durante el año 2016. Los datos y los costos directos (hospitalización y tratamiento durante la misma) fueron obtenidos de la base de datos del Dpto. de Estadísticas y del Dpto. de Ingresos del HT, respectivamente, procesados en planilla Excel.Los Años de Vida Potencialmente Perdidos (AVPP), constituyen un indicador que ilustra la pérdida que sufre la sociedad como consecuencia de la muerte de personas jóvenes o de fallecimientos prematuros. Para obtener los AVPP, se sumaron los productos del número de muertes de cada edad por la diferencia entre esta edad y una edad límite (Esperanza de vida al nacer en Paraguay en el año 2016: 73,5 años). Para describir variables categóricas se utilizó frecuencias y porcentajes, para las cuantitativas se empleó medias, desviación estándar y rangos, según corresponda.

Este estudio fue aprobado por el Comité de Ética del Hospital de Trauma. No se obtuvo consentimiento informado de los padres o tutores legales, debido a que se trata de un estudio retrospectivo basado en datos secundarios y no utilizó datos de identificación del paciente.

\section{RESULTADOS}

Se incluyeron, los 700 pacientes que acudieron al HT por accidente de moto, durante el periodo de estudio. La edad media de los pacientes fue de 15,6 \pm 4.33 años. E1 78\% (546/700) fue de sexo masculino. De los accidentados $450(64,2 \%)$ era conductor y 250 $(35,7 \%)$ acompañante. La media de edad de los conductores fue de $17 \pm 4,3$ años (rango $11-19$ años) y de los acompañantes $12,3 \pm 4,4$ (rango $<1-19$ años). Entre los conductores solo el 50.4\% (227/450) tenía la edad reglamentaria para obtener licencia de conducir. Solo el 7,7\% (54/695) de los accidentados usaba casco al momento del accidente (Tabla 1 ). 
Tabla 1. Características demográficas de los accidentados.

\begin{tabular}{lcc}
\hline Características demográficas & $\mathbf{n = 7 0 0}$ & $\%$ \\
\hline Edad media & $15,6 \pm 4.33$ & - \\
Sexo (M/F) & $546 / 154$ & $78 / 22$ \\
Conductores & 450 & 64,2 \\
$\quad$ Edad media en años \pm DE & $17 \pm 4,3$ & - \\
Acompañantes & 250 & 35,7 \\
$\quad$ Edad media en años $\pm \mathrm{DE}$ & $12,3 \pm 4,4$ & - \\
Uso de casco & 54 & 7.7 \\
\hline
\end{tabular}

El 75,7\% de los accidentados de moto pertenecían al grupo etario de 15 a 19 años; llama la atención que entre los conductores el 12\% (54/450) pertenecían al grupo de edad de 10 a 14 años (Tabla 2).

Tabla 2. Grupo de edad de los accidentados según condición de conductor o acompañante.

\begin{tabular}{cccccccc}
\hline & & \multicolumn{2}{c}{ Conductores } & & \multicolumn{2}{c}{ Acompañantes } \\
Grupo de edad & $\mathbf{n}=\mathbf{7 0 0}$ & & $\mathbf{n = 4 5 0}$ & $\mathbf{\%}$ & & $\mathbf{n}=\mathbf{2 5 0}$ & $\mathbf{\%}$ \\
\hline $0-4$ & $26(3,7)$ & 0 & 0 & & 26 & 10,4 \\
$5-9$ & $34(4,8)$ & 0 & 0 & & 34 & 13,6 \\
$10-14$ & $110(15,7)$ & 54 & 12,0 & & 56 & 22,4 \\
$15-19$ & $530(75,7)$ & 396 & 88 & & 134 & 53,6 \\
\hline
\end{tabular}

El $89,2 \%(625 / 700)$ de los pacientes requirió hospitalización por 2 o más días, de los cuales el $7.6 \%$ (48/625) requirió ser hospitalizado en la Unidad de Cuidados Intensivos Pediátricos (UCIP). La media de días de hospitalización en sala y UCIP fue de 11.04 días (DS 14,85 días) y 16.57 (DS 23 días), respectivamente.El 25,1\% (176/700) tuvo algún grado de discapacidad o secuela y el 5,4\% (38/700) falleció (Tabla 3).

Tabla 3. Requerimiento de Hospitalización y evolución de los accidentados.

\begin{tabular}{lcc}
\hline Variables & $\mathbf{n = 7 0 0}$ & $\mathbf{\%}$ \\
\hline Hospitalizados & 625 & 89.2 \\
$\quad$ En sala & 577 & 92 \\
\hline En UCIP & 48 & 7.6 \\
\hline Hospitalización (días \pm DE) & & \\
\hline En sala & $11,04 \pm 14,85$ & - \\
\hline En UCIP & $16,57 \pm 23$ & - \\
\hline Discapacidad & 176 & 25.1 \\
\hline Fallecidos & 38 & 5.4 \\
\hline
\end{tabular}

Los costos directos por hospitalización por día en sala y en UCIP fueron de 275,4 y 737,2 , respectivamente, siendo los costos directos por accidentes de moto en el año 2016 de 2.587.179,2\$ (Tabla 4), lo cual representa el $0.009 \%$ del PIB del país. Los años de vida potencialmente perdidos
(AVPP) en el año 2016, fueron de 2159años (Tabla 5).

Tabla 4. Características demográficas de los accidentados.

\begin{tabular}{lcc}
\hline $\begin{array}{l}\text { Costos médicos } \\
\text { directos }\end{array}$ & $\begin{array}{l}\text { Total de días de } \\
\text { hospitalización }\end{array}$ & Costo total (\$) \\
\hline Sala & 7635 & 2.102 .679 \\
UCIP & 668 & 492.450 \\
TOTAL & $\mathbf{8 3 0 3}$ & $\mathbf{2 . 5 9 5 . 1 2 9}$ \\
\hline
\end{tabular}

Tabla 5. Años de Vida Potencialmente Perdidos (AVPP).

\begin{tabular}{ccccc}
\hline $\begin{array}{c}\text { Grupo de edad } \\
\text { (años) }\end{array}$ & $\begin{array}{c}\text { Punto medio de } \\
\text { intervalo }\end{array}$ & $\begin{array}{c}\text { Factor de } \\
\text { ponderación }\end{array}$ & $\begin{array}{c}\text { N (\%) de } \\
\text { muertes }\end{array}$ & AVPP \\
\hline 1 a 4 & 2,5 & 70,5 & $1(2.6)$ & 70.5 \\
5 a 9 & 7,5 & 65,5 & $2(5.2)$ & 131 \\
10 a 14 & 12,5 & 60,5 & $3(7.8)$ & 181,5 \\
15 a 19 & 17,5 & 55,5 & $32(84.0)$ & 1776 \\
\hline TOTAL & & & $\mathbf{3 8}$ & $\mathbf{2 1 5 9}$ \\
\hline
\end{tabular}

\section{DISCUSIÓN}

Los traumatismos y muertes causadas por los accidentes en motocicletas constituyen un problema creciente de salud pública y afecta de forma considerable a las personas jóvenes y familias de quienes la padecen por los costos y la discapacidad asociada. Muchas de estas familias se sumen en la pobreza a consecuencia de dichos costos, que no han estimado el impacto y el sufrimiento humano que estos representan sobre todo en países de bajos y medianos ingresos.

Los motociclistas son un grupo particularmente vulnerable en la vía pública. Algunos estudios indican que el riesgo de sufrir lesiones en motocicleta es entre 10 y 30 veces superior al correspondiente de los conductores de automóviles. Esto se debe no solo a la forma del vehículo, que mantiene el cuerpo a la intemperie y limita, en comparación con los automóviles, la seguridad pasiva (como el cinturón de seguridad o la cabina del vehículo), sino que, además de la falta de protección contra choques, están expuestos a no ser vistos por otros conductores de automóviles y a los impactos con barreras de seguridad que han sido diseñadas para otro tipo de vehículos ${ }^{(1)}$.

Los resultados del presente estudio muestran que los accidentes de moto fueron más frecuentes en el 
sexo masculino (78\%), al igual que lo reportado por otros autores $^{(12-14)}$. La mayoría de los accidentados era conductor $(64,2 \%)$ con una edad media de $17 \pm 4,3$ años y con un rango de edad de 11 a 19 años. Este resultado coincide con el estudio realizado en Tailandia donde la media de la edad de accidentados de moto fue de $13.5 \pm 1.5$ años con un rango de edad de 7 años 10 meses y 14 años 11 meses $^{(14)}$.

Paraguay cuenta con una Ley $N^{\circ}$ 5016/14 Nacional de Tránsito y Seguridad Vial, que establece la edad reglamentaria para conducir, así como la obligatoriedad del uso del casco y otros elementos de protección ${ }^{(20)}$. El Informe Mundial de Seguridad de Vial de la OMS, refiere que solamente 44 países, entre los cuales se encuentra el nuestro, cuentan con leyes relativas al uso del casco que se aplican a todos los conductores y pasajeros ${ }^{(1)}$.

Sin embargo, los resultados del presente estudio, demuestran que no existe un control del cumplimiento de la mencionada Ley, por parte de los organismos municipales. Es así que solo el $50.4 \%$ (227/450) tenía la edad reglamentaria para obtener licencia de conducir y solo el 7,7\% (54/695) de los accidentados tenía casco al momento del accidente.

Existen varios estudios que demuestran el efecto protector del uso del casco, el cual puede reducir el riesgo de muerte en casi un $40 \%$ y el riesgo de sufrir traumatismos graves en aproximadamente un $70 \% \%^{(15-19)}$.

En el presente estudio, la mayoría de los accidentados (89.2\%), requirió ser hospitalizada, y $7,6 \%$ tuvo que ser ingresado a la UCIP, lo cual muestra que los accidentes fueron de moderados a severos, con una mortalidad del 5,4\% y con resultado de algún grado de discapacidad el 25,1\% (176/700).

Según un estudio sobre la carga de morbilidad global realizado por la Organización Mundial de la Salud, en el mundo, los accidentes de tránsito representaron la décima causa, entre las principales enfermedades y daños con la mayor cantidad de Años de Vida Potencialmente Perdidos en el año 1990, y alcanzó 9. 600.000 de AVPP. Mientras que en el año 2010, los accidentes de tránsito alcanzaron la quinta posición entre las 50 principales causas de mortalidad prematura, con una pérdida total de 5 . 900.000 de $\mathrm{AVPP}^{(17)}$. En el presente estudio, en el periodo de un año, los AVPP fueron 2159, en $\leq 19$ años y solo por accidentes de moto.

Este estudio, solo reporta los costos directos por accidentes de moto en $\leq 19$ años, sin embargo, los mismos alcanzan un total de $\$ 2.595 .129$, solo en el periodo de un año. Por otro lado, el hecho de que la mayoría de los accidentados es del sexo masculino y si esta tendencia actual continúa, más jóvenes adultos en el grupo económicamente productivo seguirán muriendo o quedando con discapacidades como resultado de un accidente de moto. Este hecho reduce la productividad en este grupo poblacional y puede tener enormes consecuencias económicas negativas para las familias y para el país en su conjunto.

Los accidentes en moto no solo representan una carga importante en la morbilidad y mortalidad a la que se enfrenta el sistema de salud sino que, impactan negativamente desde el punto de vista económico, debido al volumen de recursos financieros, materiales y humanos que demandan dentro y fuera del sector salud, para su control, tratamiento y rehabilitación.

A pesar de las limitaciones del estudio al no incluir costos indirectos y costos intangibles como pena, sufrimiento, pérdida de trabajo, entre otros; constituye el primer estudio de costos por accidentes de moto en la edad pediátrica realizado en nuestro país. Además proporciona información útil sobre la carga de los accidentes de este tipo, la cual puede ser utilizada como referencia para llevar a cabo otras investigaciones y diseño de programas de intervención. Así mismo podría orientar a intervenciones efectivas de salud pública para reducir significativamente la carga de por accidentes de tránsito. Finalmente, los resultados del presente trabajo, constituyen un sólido fundamento para que los organismos municipales de control, definan acciones estratégicas para el cumplimiento de la Ley $\mathrm{N}^{\circ} 5016 / 14^{(20)}$.

\section{CONCLUSIONES}

Los accidentes de motos fueron más frecuentes en el 
sexo masculino; la mayoría requirió ser hospitalizado, y se observó una mortalidad y discapacidad elevadas. El impacto de las altas tasas de mortalidad y discapacidad, así como de los AVPP

\section{REFERENCIAS}

1. OMS. Lesiones causadas por el tránsito. (Internet). OMS; 2018. Citado el 19 de febrero de 2018. Recuperado a partir de: http://bit.ly/19zLNCQ

2. OMS. Diez estrategias para preservar la seguridad de los niños en las carreteras. OMS; 2015.

3. World Health Organization; UNICEF. World Report on Child Injury Prevention. Summary. Geneva: WHO;2008.

4. Miranda J, Huicho L, López Rivera LA, Paca A, Luna D, Rosales $\mathrm{E}$, et al. Lesiones más frecuentes asociadas a accidentes de tránsito; severidad, letalidad y factores asociados a esta severidad y letalidad. Lima; 2009.

5. United Nations. Sixty-Second General Assembly Plenary 87th Meeting. New York: United Nations; 2008. (General Assembly Adopts Resolution on Easing Global Road Safety Crisis).

6. Peden M, McGee K, Sharma G. The Injury Chart Book: A Graphical Overview of the Global Burden of Injuries. Geneva: World Health Organization; 2002.

7. García-Altés A, Pérez K. The economic cost of road traffic crashes in an urban setting. Inj Prev 2007; 16:65-8.

8. Marracino C, Royer ME, Irurzun A, García V, Capentti R. Programa VIGIA Ministerio de Salud de la Nación: carga global de enfermedad e impacto económico por accidentes de transporte ocurridos en la ciudad de Buenos Aires. 2003.

9. Agencia Nacional de Tránsito y Seguridad Vial. Informe estadístico del observatorio vial: registro de datos Dra. Mercedes Maldonado Banks. ANTSV;2015.

10. MSPyBS. Datos del Departamento de Estadísticas: Hospital Manuel Giagni, MSPyBS y Dpto de Ingresos. Paraguay: MSPyBS; 2016.

11. Organización Panamericana de la salud. Cascos: manual de seguridad vial para decisores y profesionales. Washington: OPS, 2008. (Publicación Científica y Técnica no 628). por las lesiones causadas por accidentes de moto en $\leq 19$ años, solo en un año, representa un costo económico y social extremo derivado de una causa de muerte prevenible.

12. Berrones-Sanz, LD. Análisis de los accidentes y las lesiones de los motociclistas en México. Gac Med Mex. 2017;153:662-671

13. Mendonça MFD, Santana Cabral SilvaI AC, Lima de Castro CC. Análise espacial dos acidentes de trânsito urbano atendidos pelo Serviço de AtendimentoMóvel de Urgência: um recorte no espaço e no tempo. Rev Bras Epidemiol Our-Dez; 20(4): 727-741.

14. Tunthanathip T, Phuenpathom N. Impact of road traffic injury to pediatric traumatic brain injury in Southern Thailand. J Neurosci Rural Pract 2017;8:601-8

15. GBD 2013 Mortality and Causes of Death Collaborators. Global, regional, and national age-sex specific all-cause and cause-specific mortality for 240 causes of death, 1990-2013: a systematic analysis for the Global Burden of Disease Study 2013. Lancet. 2015 January 10;385(9963): 117-171.

16. Olson Z, Staples JA, Mock C, Nguyen NP, Bachani AM, Nugent R, et al. Helmet regulation in Vietnam: impact on health, equity and medical impoverishment. Prev. 2016 Aug;22(4):233-8.

17. Adams NS, Newbury PA, Eichhorn MG, Davis AT, Mann RJ, Polley JW, et al. The Effects of Motorcycle Helmet Legislation on Craniomaxillofacial Injuries. Plast Reconstr Surg. 2017 Jun; 139(6):1453-1457.

18. Khor D, Inaba K, Aiolfi A, Delapena S, Benjamin E, Matsushima K, et al. The impact of helmet use on outcomes after a motorcycle crash. Injury. 2017 May;48(5):1093-1097.

19. Gupta S, Iv V, Sam N, Vuthy D, Klaric K, Shrime MG, et al. Impact of Helmet Use on Severity of Epidural Hematomas in Cambodia. World Neurosurgery. 2017 Apr; 100:267-270.

20. Congreso de la Nación Paraguaya. Ley № 5016: Nacional de Transito y Seguridad Vial. 2014. 